DOI: $10.15393 /$ j3.art.2022.10770

UDC 517.1, 517.521

R. M. Dhaigude, Y. J. Bagul

\title{
A NOTE ON THE BECKER-STARK TYPE INEQUALITIES
}

\begin{abstract}
This note is devoted to establishing the sharp bounds for the function $x / \operatorname{tg} x$, thus refining the well-known Becker-Stark's inequality.
\end{abstract}

Key words: Becker-Stark inequality, tangent function, monotonicity of functions, Bernoulli numbers

2020 Mathematical Subject Classification: 26A48, 26D05, $33 B 10$

1. Introduction. The inequality

$$
1-\frac{4 x^{2}}{\pi^{2}}<\frac{x}{\operatorname{tg} x}<\frac{\pi^{2}}{8}-\frac{x^{2}}{2} ; \quad x \in(0, \pi / 2)
$$

is known in the literature as the Becker-Stark inequality. Here tg denotes the trigonometric tangent function. It was proved in [6]. Z.-H. Yang et. al. in [15] prove that

$$
1-\frac{4 x^{2}}{\pi^{2}}<\frac{x}{\operatorname{tg} x}<1-\frac{x^{2}}{3} ; \quad x \in(0, \pi / 2),
$$

while Chen and Cheung [7] show that

$$
\left(1-\frac{4 x^{2}}{\pi^{2}}\right)<\frac{x}{\operatorname{tg} x}<\left(1-\frac{4 x^{2}}{\pi^{2}}\right)^{\pi^{2} / 12} ; x \in(0, \pi / 2)
$$

with the best possible constants 1 and $\pi^{2} / 12$. The lower bound in all the three inequalities listed above is one and the same. However, the upper bound in (3) is sharper than those in (1) and (2). The upper bounds in (2)-(3) are not sharp as $x \rightarrow \pi / 2^{-}$. Researchers obtained different

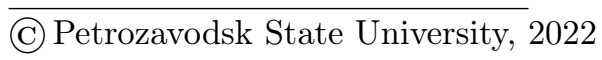


generalizations and refinements of inequality (1). The details can be seen in [3-10], [13], [15-18] and the references therein.

In view of obtaining refinement of the lower bounds in inequalities (1) - (3) and the sharp upper bound for $x / \operatorname{tg} x$ as $x \rightarrow \pi / 2^{-}$, we propose the following theorem:

Theorem 1. For $x \in(0, \pi / 2)$, we have

$$
\left(1-\frac{4 x^{2}}{\pi^{2}}\right) e^{\left(\frac{4}{\pi^{2}}-\frac{1}{3}\right) x^{2}}<\frac{x}{\operatorname{tg} x}<\left(1-\frac{4 x^{2}}{\pi^{2}}\right) e^{\frac{4 \ln \left(\pi^{2} / 8\right)}{\pi^{2}} x^{2}} .
$$

The bounds in (4) are polynomial-exponential in nature. Since $\left(\frac{4}{\pi^{2}}-\frac{1}{3}\right) x^{2}>1$, the lower bound in (4) is sharper than the one in (1) - (3). With the help of any plotting software, it can be observed that the upper bound in (4) is sharper than the corresponding upper bound in (3) for $x \in\left(\xi_{1}, \pi / 2\right)$, where $\xi_{1} \approx 0.8496$. The constant $\left(\frac{4}{\pi^{2}}-\frac{1}{3}\right)$ (or a similar) one is interesting, and as a complement to (4), we present simple polynomial bounds for $x / \tan x$ as the following double inequality:

Proposition 1. For $x \in(0, \pi / 2)$, we have

$$
\left(1-\frac{4 x^{2}}{\pi^{2}}\right)\left[1+\left(\frac{4}{\pi^{2}}-\frac{1}{3}\right) x^{2}\right]<\frac{x}{\operatorname{tg} x}<\left(1-\frac{4 x^{2}}{\pi^{2}}\right)\left[1-\left(\frac{4}{\pi^{2}}-\frac{1}{2}\right) x^{2}\right] .
$$

As $1+\left(\frac{4}{\pi^{2}}-\frac{1}{3}\right) x^{2}>1$, we conclude that the lower bound in (5) is sharper than the one in (1)-(3). And again, with the help of any plotting software, one can observe that the upper bound in (5) is sharper than the corresponding upper bound in (3) for $x \in\left(\xi_{2}, \pi / 2\right)$, where $\xi_{2} \approx 0.9721$.

2. Preliminaries and lemmas. We recall the formula for the simple geometric series:

$$
\frac{1}{1-x}=1+x+x^{2}+x^{3}+\ldots,|x|<1
$$

and

$$
\frac{x}{\sin x}=1+\sum_{k=1}^{\infty} \frac{2\left(2^{2 k-1}-1\right)}{(2 k) !}\left|B_{2 k}\right| x^{2 k}, \quad|x|<\pi,
$$

where $B_{2 k}$ are the even indexed Bernoulli numbers. The expansion (7) can be found in $[11,1.411]$. We also need the following lemmas for proving our main results. The Lemma 1 is known as l'Hôpital's rule of monotonicity. We refer to [2] for more details. 
Lemma 1. Let $f_{1}(x)$ and $f_{2}(x)$ be two real-valued functions that are continuous on $[a, b]$ and differentiable on $(a, b)$, where $-\infty<a<b<\infty$ and $g^{\prime}(x) \neq 0$, for all $x \in(a, b)$. Let

$$
\begin{aligned}
& A(x)=\frac{f_{1}(x)-f_{1}(a)}{f_{2}(x)-f_{2}(a)}, x \in(a, b), \\
& B(x)=\frac{f_{1}(x)-f_{1}(b)}{f_{2}(x)-f_{2}(b)}, x \in(a, b) .
\end{aligned}
$$

Then we have

(i) $A(x)$ and $B(x)$ are increasing on $(a, b)$ if $f_{1}^{\prime}(x) / f_{2}^{\prime}(x)$ is increasing on $(a, b)$.

(ii) $A(x)$ and $B(x)$ are decreasing on $(a, b)$ if $f_{1}^{\prime}(x) / f_{2}^{\prime}(x)$ is decreasing on $(a, b)$.

The strictness of the monotonicity of $A(x)$ and $B(x)$ depends on the strictness of monotonicity of $f_{1}^{\prime}(x) / f_{2}^{\prime}(x)$.

Lemma 2. For all integers $k \geqslant 1$, we have

$$
\left|B_{2 k}\right|<\frac{2(2 k) !}{(2 \pi)^{2 k}} \frac{1}{1-2^{\beta-2 k}},
$$

where $\beta=2+\left(\ln \left(1-6 / \pi^{2}\right)\right) / \ln 2 \approx 0.6491$.

Lemma 2 appears in [1] and Lemma 3 is proved by L. Zhu et.al. in [17].

Lemma 3. For $|x|<\pi / 2$, we have

$$
\left(\pi^{2}-4 x^{2}\right) \frac{\operatorname{tg} x}{x}=\pi^{2}+\sum_{k=1}^{\infty} a_{k} x^{2 k}
$$

where $a_{k}=\frac{2^{2 k+2}\left(2^{2 k+2}-1\right) \pi^{2}}{(2 k+2) !}\left|B_{2 k+2}\right|-\frac{4 \cdot 2^{2 k}\left(2^{2 k}-1\right)}{(2 k) !}\left|B_{2 k}\right|<0$, $k=1,2,3, \ldots$

3. Proofs of the main results. In this section, we prove our main results.

Proof of Theorem 1. Consider

$$
f(x)=\ln \left(\frac{x}{\operatorname{tg} x} \frac{\pi^{2}}{\pi^{2}-4 x^{2}}\right) / x^{2}=\frac{f_{1}(x)}{f_{2}(x)},
$$


where $f_{1}(x)=\ln \left(\frac{x}{\operatorname{tg} x} \frac{\pi^{2}}{\pi^{2}-4 x^{2}}\right)$ and $f_{2}(x)=x^{2}$ with $f_{1}(0+)=0$ and $f_{2}(0)=0$. Differentiation of the numerator and denominator with respect to $x$ gives

$$
\begin{aligned}
& \frac{f_{1}^{\prime}(x)}{f_{2}^{\prime}(x)}=\frac{\frac{\operatorname{tg} x-x \sec ^{2} x}{x \operatorname{tg} x}+\frac{8 x}{\pi^{2}-4 x^{2}}}{2 x}= \\
& =\frac{1}{2} \frac{\operatorname{tg} x-x \sec ^{2} x}{x^{2} \operatorname{tg} x}+\frac{4}{\pi^{2}-4 x^{2}}= \\
& =\frac{1}{4} \frac{\sin 2 x-2 x}{x^{2} \sin 2 x}+\frac{4}{\pi^{2}} \frac{1}{\left(1-\frac{4 x^{2}}{\pi^{2}}\right)}= \\
& =\frac{1}{4 x^{2}}-\frac{1}{4 x^{2}} \cdot \frac{2 x}{\sin 2 x}+\frac{4}{\pi^{2}} \cdot \frac{1}{\left[1-\left(\frac{2 x}{\pi}\right)^{2}\right]} \text {. }
\end{aligned}
$$

Using (6) and (7), we write

$$
\begin{aligned}
\frac{f_{1}^{\prime}(x)}{f_{2}^{\prime}(x)} & =\frac{1}{4 x^{2}}-\frac{1}{4 x^{2}}\left[1+\sum_{k=1}^{\infty} \frac{2^{2 k+1}\left(2^{2 k-1}-1\right)}{(2 k) !}\left|B_{2 k}\right| x^{2 k}\right]+\frac{4}{\pi^{2}} \sum_{k=0}^{\infty}\left(\frac{2 x}{\pi}\right)^{2 k}= \\
& =\frac{4}{\pi^{2}} \sum_{k=0}^{\infty} \frac{2^{2 k}}{\pi^{2 k}} x^{2 k}-\sum_{k=1}^{\infty} \frac{2^{2 k-1}\left(2^{2 k-1}-1\right)}{(2 k) !}\left|B_{2 k}\right| x^{2 k-2}= \\
& =\sum_{k=0}^{\infty} \frac{2^{2 k+2}}{\pi^{2 k+2}} x^{2 k}-\sum_{k=0}^{\infty} \frac{2^{2 k+1}\left(2^{2 k+1}-1\right)}{(2 k+2) !}\left|B_{2 k+2}\right| x^{2 k}= \\
& =\sum_{k=0}^{\infty} 2^{2 k+1}\left[\frac{2}{\pi^{2 k+2}}-\frac{\left(2^{2 k+1}-1\right)}{(2 k+2) !}\left|B_{2 k+2}\right|\right] x^{2 k}:=\sum_{k=0}^{\infty} c_{k} x^{2 k}
\end{aligned}
$$

where $c_{k}=2^{2 k+1}\left[\frac{2}{\pi^{2 k+2}}-\frac{\left(2^{2 k+1}-1\right)}{(2 k+2) !}\left|B_{2 k+2}\right|\right]$.

From (8), we have

$$
\left|B_{2 k+2}\right|<\frac{2(2 k+2) !}{2^{2 k+2} \pi^{2 k+2}} \frac{1}{1-2^{\beta-2 k-2}}=\frac{2(2 k+2) !}{\pi^{2 k+2}} \frac{1}{\left(2^{2 k+2}-2^{\beta}\right)},
$$

where $\beta=2+\left(\ln \left(1-6 / \pi^{2}\right)\right) / \ln 2 \approx 0.6491$. 
Clearly, $2^{\beta}-1<2^{2 k+1}$ for $k \geqslant 0$, i. e., $2^{\beta}-1<2^{2 k+2}-2^{2 k+1}$ or $2^{2 k+1}-1<2^{2 k+2}-2^{\beta}$. From this, we write:

$$
\frac{1}{2^{2 k+2}-2^{\beta}}<\frac{1}{2^{2 k+1}-1} .
$$

Then

$$
\left|B_{2 k+2}\right|<\frac{2(2 k+2) !}{\pi^{2 k+2}} \frac{1}{\left(2^{2 k+2}-2^{\beta}\right)}<\frac{2(2 k+2) !}{\pi^{2 k+2}} \frac{1}{\left(2^{2 k+1}-1\right)},
$$

which leads to $c_{k}>0$ for $k \geqslant 0$. Hence, $f_{1}^{\prime}(x) / f_{2}^{\prime}(x)$ is strictly increasing on $(0, \pi / 2)$. By Lemma 1, $f(x)$ is also strictly increasing on $(0, \pi / 2)$. So, $f(0+)<f(x)<f(\pi / 2-)$. The limits $f(0+)=4 / \pi^{2}-1 / 3$ and $f(\pi / 2-)=4 \ln \left(\pi^{2} / 8\right) / \pi^{2}$ prove the assertion.

Proof of Proposition 1. Let

$$
g(x)=\left(\frac{\pi^{2}}{\frac{\operatorname{tg} x}{x}\left(\pi^{2}-4 x^{2}\right)}-1\right) / x^{2}, x \in(0, \pi / 2) .
$$

By Lemma 3, we get

$$
g(x)=\left(\frac{\pi^{2}}{\pi^{2}+\sum_{k=1}^{\infty} a_{k} x^{2 k}}-1\right) / x^{2}=\frac{-\sum_{k=1}^{\infty} a_{k} x^{2 k}}{\pi^{2} x^{2}+\sum_{k=1}^{\infty} a_{k} x^{2 k+2}},
$$

where $a_{k}<0$ for $k=1,2,3, \ldots$ Then

$$
\frac{1}{g(x)}=\frac{\pi^{2} x^{2}+\sum_{k=1}^{\infty} a_{k} x^{2 k+2}}{-\sum_{k=1}^{\infty} a_{k} x^{2 k}}=\frac{-\pi^{2}}{\sum_{k=1}^{\infty} a_{k} x^{2 k-2}}-x^{2} .
$$

This implies that $g(x)$ is strictly increasing on $(0, \pi / 2)$. So, we have $g(0+)<g(x)<g(\pi / 2-)$. With the limits $g(0+)=4 / \pi^{2}-1 / 3$ and $g(\pi / 2-)=1 / 2-4 / \pi^{2}$, we end the proof.

A graphical illustration of the lower and upper bounds of $x / \operatorname{tg} x$ appeared in (1)-(5) is given in Figure 1 and Figure 2. 


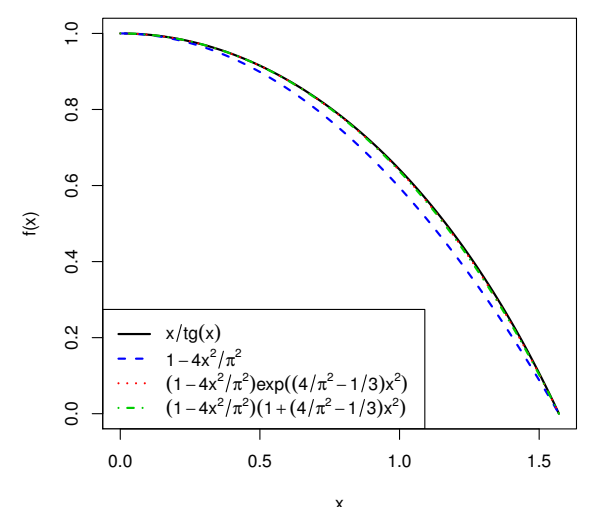

Figure 1: Graphs of the lower bounds of $(1)-(5), x \in(0, \pi / 2)$.

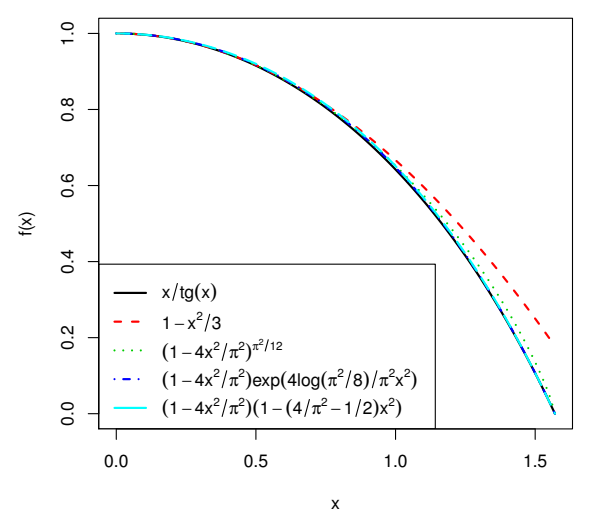

Figure 2: Graphs of the upper bounds of $(2)-(5), x \in(0, \pi / 2)$.

\section{Extended inequalities via monotonically stratified func-} tions. We extend inequalities (4) and (5) to a wider range of parameters using the technique of the minimax approximant given in [12]. For this, consider the family of continuous functions

$$
\phi_{p}(x)=\frac{x}{\sin x}-\left(1-\frac{4 x^{2}}{\pi^{2}}\right) e^{p x^{2}} \sec x
$$

on $(0, \pi / 2)$ for every $p \in \mathbf{R}^{+}$. Clearly, the family of functions $\phi_{p}(x)$ is decreasingly stratified for $p \in \mathbf{R}^{+}$. If

$$
A=\frac{4}{\pi^{2}}-\frac{1}{3}=0.071951 \ldots \text { and } B=\frac{4 \ln \left(\pi^{2} / 8\right)}{\pi^{2}}=0.085117 \ldots,
$$

then we have $\phi_{A}(0+)=\phi_{B}(0+)=\phi_{B}(\pi / 2-)=0$ and $\phi_{A}(\pi / 2-) \in \mathbf{R}^{+}$. Moreover, the functions $\phi_{p}(x)$ are continuous with respect to $p \in(A, B)$ for every $x \in(0, \pi / 2)$ and $\phi_{p}(\pi / 2-)$ is continuous with respect to $p \in(A, B)$. So, using Theorem 1' and Theorem 2' of [12], we have the following statements:

Statement 1. If $p \in(0, A]$, where $A=\frac{4}{\pi^{2}}-\frac{1}{3}=0.071951 \ldots$, then

$$
\phi_{p}(x) \geqslant \phi_{A}(x)=\frac{x}{\sin x}-\left(1-\frac{4 x^{2}}{\pi^{2}}\right) e^{\left(\frac{4}{\pi^{2}}-\frac{1}{3}\right) x^{2}} \sec x>0
$$

for $x \in(0, \pi / 2)$. 
Statement 2. If $p \in[B, \infty)$, where $B=\frac{4 \ln \left(\pi^{2} / 8\right)}{\pi^{2}}=0.085117 \ldots$, then

$$
\phi_{p}(x) \leqslant \phi_{B}(x)=\frac{x}{\sin x}-\left(1-\frac{4 x^{2}}{\pi^{2}}\right) e^{\frac{4 \ln \left(\pi^{2} / 8\right)}{\pi^{2}} x^{2}} \sec x<0
$$

for $x \in(0, \pi / 2)$.

Similarly, if we consider

$$
\psi_{p}(x)=\frac{x}{\sin x}-v\left(1-\frac{4 x^{2}}{\pi^{2}}\right)\left(1+p x^{2}\right) \sec x, p \in \mathbf{R}^{+}
$$

and

$$
C=\frac{4}{\pi^{2}}-\frac{1}{3}=0.071951 \ldots, D=\frac{1}{2}-\frac{4}{\pi^{2}}=0.094715 \ldots
$$

then it is again obvious that the family of functions $\psi_{p}(x)$ is decreasingly stratified and satisfies the assumptions of Theorem 1' of [12]. So, using Theorem 2' of [12], we state the following:

Statement 3. If $p \in(0, C]$ where $C=\frac{4}{\pi^{2}}-\frac{1}{3}=0.071951 \ldots$, then

$$
\psi_{p}(x) \geqslant \psi_{C}(x)=\frac{x}{\sin x}-\left(1-\frac{4 x^{2}}{\pi^{2}}\right)\left[1+\left(\frac{4}{\pi^{2}}-\frac{1}{3}\right) x^{2}\right] \sec x>0
$$

for $x \in(0, \pi / 2)$.

Statement 4. If $p \in[D, \infty)$, where $D=\frac{1}{2}-\frac{4}{\pi^{2}}=0.094715 \ldots$, then

$$
\psi_{p}(x) \leqslant \psi_{D}(x)=\frac{x}{\sin x}-\left(1-\frac{4 x^{2}}{\pi^{2}}\right)\left[1+\left(\frac{1}{2}-\frac{4}{\pi^{2}}\right) x^{2}\right] \sec x
$$

for $x \in(0, \pi / 2)$.

Acknowledgement. The authors would like to thank the referees for their valuable comments and suggestions.

\section{References}

[1] Alzer H. Sharp bounds for the Bernoulli numbers. Archiv der Mathematik, 2000, vol. 74, no. 3, pp. 207-211.

[2] Anderson G. D., Vamanamurthy M. K., Vuorinen M. Conformal Invariants, Inequalities and Quasiconformal Maps. John Wiley and Sons, New York, 1997. 
[3] Bagul Y. J., Chesneau C. New sharp bounds for tangent function. Bull. Allahabad Math. Soc., 2019, vol. 34, no. 2, pp. 277-282.

[4] Bagul Y. J., Kostić M., Chesneau C., Dhaigude R. M. On the generalized Becker-Stark type inequalities. Acta Univ. Sapientiae Math., 2021, vol. 13, no. 1 , pp. $92-108$.

DOI: https://doi.org/10.2478/ausm-2021-0005

[5] Banjac B., Makragić M., Malešević B. Some notes on a method for proving inequalities by computer. Results Math., 2016, vol. 69, no. 1, pp. 161-176.

[6] Becker M., Stark E. L. On a hierarchy of quolynomial inequalities for $\tan x$. Univ., Beograd. Publ. Elektrotehn. Fak. Ser. Mat. Fiz., 1978, no. 602/633, pp. $133-138$.

[7] Chen C.-P., Cheung W.-S. Sharp Cusa and Becker-Stark inequalities. J. Inequal. Appl., 2011, 2011:136.

DOI: https://doi.org//10.1186/1029-242X-2011-136

[8] Chen C.-P., Elezović N. Sharp Redheffer-type and Becker-Stark-type inequalities with an application. Math. Inequal. Appl., 2018, vol. 21, no. 4, pp. $1059-1078$.

[9] Chen C.-P., Sándor J. Sharp inequalities for trigonometric and hyperbolic functions. J. Math. Inequal., 2015, vol. 9, no. 1, pp. 203-217.

[10] Debnath L., Mortici C., Zhu L. Refinements of Jordan-Stečkin and BeckerStark inequalities. Results. Math., 2015, vol. 67, pp. 207-215.

[11] Gradshteyn I. S., Ryzhik I. M. Table of Integrals, Series and Products. Elsevier, Seventh Edition, 2007.

[12] Malešević B., Mihailović B. A minimax approximant in the theory of analytic inequalities. Appl. Anal. Discrete Math., 2021, vol. 15, no. 2, pp. 486 -509. DOI: https://doi.org/10.2298/AADM210511032M

[13] Nishizawa Y. Sharp Becker-Stark's type inequalities with power exponential functions. J. Inequal. Appl., 2015, 402(2015).

DOI: https://doi.org/10.1186/s13660-015-0932-9

[14] Wu Y., Bercu G. New refinements of Becker-Stark and Cusa-Huygens inequalities via trigonometric polynomials method, RACSAM Rev. R. Acad. Cienc. Exactas Fís. Nat. Ser. A Mat., 115, 87(2021).

DOI: https://doi.org/10.1007/s13398-021-01030-6

[15] Yang Z.-H., Jiang Y.-L., Song Y.-Q., Chu Y.-M. Sharp inequalities for trigonometric functions. Abstr. Appl. Anal., 2014, Article ID 601839, 18 p.

[16] Zhu L. Simple proofs of the Cusa-Huygens-type and Becker-Stark-type inequalities. J. Math. Inequal., 2013, vol. 7, no. 4, pp. 563-567. 
[17] Zhu L., Hua J.-K. Sharpening the Becker-Stark inequalities. J. Inequal. Appl., 2010, Article ID 931275.

[18] Zhu L. A refinement of the Becker-Stark inequalities. Math. Notes, 2013, vol. 93 , pp. $421-425$.

DOI: https://doi.org/10.1134/S0001434613030085

Received August 17, 2021.

In revised form, January 09, 2022.

Accepted January 24, 2022.

Published online February 3, 2022.

Ramkrishna M. Dhaigude

Department of Mathematics

Government Vidarbha Institute of Science and Humanities

Amravati(M. S.)-444604, India

E-mail: rmdhaigude@gmail.com

Yogesh J. Bagul

Department of Mathematics

K. K. M. College, Manwath

Dist: Parbhani(M. S.)-431505, India

E-mail: yjbagul@gmail.com 